\title{
Uma nova abordagem para a medição da diluição de soldagem, baseada nos pontos de inflexão de um Contorno Ativo
}

\author{
A new approach for the measurement of \\ welding dilution, based on inflection \\ points of an Active Contours
}

Gabriel Bandeira Holanda ${ }^{1}$, Daniel de Alencar Lima ${ }^{1}$, Pedro Pedrosa Rebouças Filho ${ }^{1}$

\footnotetext{
${ }^{1}$ Laboratório de Processamento Digital de Imagens e Simulação Computacional (LAPISCO), Instituto Federal de Educação, Ciência e Tecnologia do Ceará (IFCE), Maracanaú, Ceará, Brasil. e-mail: gabrielbandeira@lapisco.ifce.edu.br, danielalencar@lapisco.ifce.edu.br, pedrosarf@ifce.edu.br
}

\section{RESUMO}

Em diversas aplicações, com ênfase nos processos de soldagem dissimilar, a diluição, em conjunto com outros fatores, pode afetar as propriedades mecânicas dos materiais soldados, sendo assim, capaz de gerar falhas durante as condições normais de serviço. Deste modo, a taxa de diluição geralmente é calculada a partir da análise de imagens digitais, com o auxílio de programas de desenho que determinam manualmente a área de penetração e a área total do metal de solda. Entretanto, este processo de cálculo é caracterizado por ser exaustivo e propenso ao erro devido a fadiga do operador. Assim, com o intuito de reduzir o tempo de análise e eliminar o máximo possível de erros no cálculo, este trabalho propõe uma nova abordagem para determinação da taxa de diluição do metal de solda sobre o metal de base. Para isto, a região da solda é determinada a partir do Método de Contornos Ativos Balão. Em sequência, os pontos que definem a linha de separação entre a região do metal de base fundido e a região com metal de enchimento são determinados automaticamente por meio do cálculo dos pontos de inflexão da curva. No fim, a taxa de diluição é calculada considerando as áreas segmentadas e devidamente destacadas pela linha de separação. Testes são realizados e comparados com o resultado obtido por especialistas utilizando o sistema tradicional a fim de avaliar a abordagem proposta. Os resultados obtidos foram satisfatórios para a aplicação, pois a abordagem proposta apresentou rapidez e precisão superior aos métodos encontrados na literatura.

Palavras-chave: Soldagem, Diluição, Método de Contornos Ativos Balão, Inflexão.

\begin{abstract}
In several applications with dissimilar welding processes, the dilution can affect the mechanical properties of welded materials, because this method enables the occurrence of faults during normal service conditions. The dilution rate usually is calculated from the digital images analysis, aided by drawing programs that manually determine the areas of base metal melted and the weld metal region. However, this calculation process is characterized by being an exhaustive and error-prone due to operator fatigue. Thus, to reduce the analysis time and eliminate possible errors, this work proposes a new approach for the automatic determination of dilution rate. For this, the welded region is determined by the Balloon Active Contour Method. Then, the points that describe the separation line between the regions of base metal melted and filler metal added are determined automatically by calculating the inflection points of the Active Contour. Finally, the dilution rate is calculated considering the segmented areas properly highlighted by separation line. Tests are performed and compared to the results obtained by specialists using the traditional system, to evaluate the proposed approach. The results obtained were satisfactory for the application, as the proposed approach presented superior speed and precision than the methods found in the literature.
\end{abstract}

Keywords: Welding, Dilution, Balloon Active Contour Method, Inflection. 


\section{INTRODUÇÃO}

As técnicas de Processamento Digital de Imagens (PDI) possuem diversas aplicações na área de análise de materiais, tais como, caracterização microestrutural [1, 2, 3, 4], determinação da densidade de nódulos de grafita [5, 6] e na análise dos ferros fundidos $[7,8,9,10]$.

Dentre as diversas outras análises de materiais capazes de serem realizadas por técnicas de PDI, pode ser citada a avaliação das propriedades de uma junta soldada. Os principais aspectos que afetam as características e propriedades do metal de solda são a diluição, a volatilização, as reações químicas, a absorção de gases e a estrutura de solidificação [11]. Neste trabalho, focou-se na análise da taxa de diluição.

Geralmente, na soldagem de união em aços estruturais e em aços de construção mecânica de baixo carbono não é originado qualquer tipo de problema devido ao valor da diluição, pois os consumíveis utilizados sejam varetas, arames ou eletrodos, são totalmente similares ao metal de base. Porém, quando a soldagem é dissimilar, ou seja, um dos materiais de base ou o consumível apresenta composição diferente dos demais, podem começar a existir problemas de elevada diluição [11].

Uma das principais aplicações de soldagem dissimilar consiste em revestimentos contra corrosão ou contra desgaste. No caso da soldagem de revestimentos o consumível é quase sempre de composição diferente do metal de base e os elementos de liga presentes são bem balanceados para garantir o efeito desejado. Considerando que o metal de base normalmente é de aço carbono fica claro que se houver uma diluição elevada do metal de base, o depósito pode não favorecer a resistência necessária [12].

Outra aplicação de soldagem dissimilar ocorre quando são utilizados metais de base diferentes devido à necessidade de uma característica específica em uma dada região do equipamento como, por exemplo, em uma caldeira onde em baixa temperatura se utilizam tubos de aço carbono, enquanto regiões mais quentes exigem aços ao cromo-molibdênio ou mesmo aços inoxidáveis austeníticos (de alto teor de carbono) [12].

A taxa de diluição, em geral, é computada manualmente com o auxílio de programas de desenho através da determinação da área do metal de base fundida e da área total do metal de solda.

Para auxiliar a solução deste problema, algumas ferramentas que utilizam técnicas de PDI foram desenvolvidas. Usualmente, na etapa de segmentação são utilizadas variações do Método de Contornos Ativos (MCA) para definição das regiões de interesse que servem como base para cálculo da diluição.

Em REBOUÇAS et al. [13] foi utilizado o método MCA Greedy. Já em RIBEIRO et al. [14] foi utilizado o método MCA Balão. Entretanto, apesar da etapa de segmentação se dar de forma automática, em ambos os trabalhos, os pontos que definem a linha de separação entre a região do metal de base fundido e a região do metal de enchimento são determinados manualmente.

Este trabalho propõe uma nova abordagem capaz de determinar, de forma automática, o valor da taxa de diluição do metal de solda sobre o metal de base utilizando técnicas de Processamento Digital de Imagens. Além disso, a determinação dos pontos que definem a linha de separação, foi realizada automaticamente através de uma nova abordagem que calcula os pontos de inflexão a partir da curva resultante do Método de Contornos Ativos Balão.

\section{MATERIAIS E MÉTODOS}

Nesta seção são descritas todas as etapas do sistema computacional desenvolvido, que realiza automaticamente a medição da taxa de diluição através de imagens digitais. A Figura 1, apresenta o resumo de todas as etapas deste sistema.

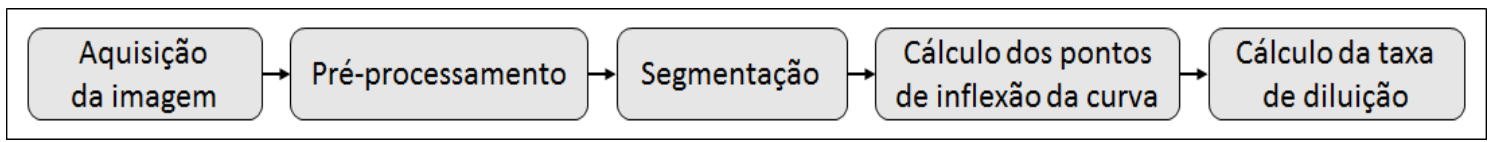

Figura 1: Diagrama de blocos do sistema desenvolvido.

As subseções seguintes vão descrever individualmente os passos adotados para explicar detalhadamente o sistema proposto.

\subsection{Aquisição da imagem e pré-processamento}

As imagens utilizadas neste trabalho foram obtidas por meio de um estereoscópio, e as amostras envolveram 
a soldagem de diferentes tipos de aços com depósito simples.

Geralmente, a etapa de pré-processamento consiste em melhorar a qualidade da disposição dos dados na imagem digital, visando aumentar o acerto no processo de segmentação [15]. Portanto, algoritmos são utilizados para reduzir regiões indesejáveis na imagem, oriundas da etapa de aquisição. Inicialmente é realizada a conversão da imagem capturada pelo estereoscópio no formato RGB para tons de cinza. Deste modo, torna-se o sistema proposto mais rápido devido a diminuição da quantidade de dados a serem analisados. Em seguida, é aplicado o filtro passa-baixa da Mediana, configurado com uma matriz de ordem 5, que substitui o nível de cinza de cada pixel pela mediana dos níveis de cinza dos vizinhos, isso implica na atenuação dos ruídos com mínima perda de informação das bordas [15].

\subsection{Segmentação}

A segmentação subdivide uma imagem em suas partes ou objetos constituintes [15]. As técnicas de segmentação são classificadas em relação a duas propriedades básicas: similaridade e descontinuidade. A primeira é a divisão da imagem em regiões não similares, em que o critério de similaridade é pré-definido. Já a descontinuidade separa as regiões da imagem com base em modificações nas características dos pixels vizinhos.

Os métodos de segmentação que atualmente demonstraram excelente aplicabilidade em imagens de diluição na soldagem, são os Métodos de Contornos Ativos (MCA's), conhecido do inglês Snakes. Os MCA's, são utilizados para segmentar objetos de interesse da imagem a partir de um contorno inicial, que se deforma através da minimização iterativa de uma função que representa a energia do contorno. O estudo referente aos MCA's foi introduzido por KASS et al. [16]. A função energia que representa esse modelo deformável é descrita pela seguinte equação:

$$
E=\int_{0}^{1}\left[e_{1}\left|c^{\prime}(s)^{2}\right|+e_{2}\left|c^{\prime \prime}(s)^{2}\right|+E_{e x t}(c(s))\right] d s,
$$

A função energia $E$ é composta por uma energia interna e por uma energia externa $\left(E_{\text {ext }}\right)$. A energia interna é definida pelas derivações da função $c(s)$, em que a primeira derivada $c^{\prime}(s)$ é dita como energia de elasticidade do contorno, que traduz a sua capacidade de se distanciar ou se aproximar dos pontos vizinhos. A segunda derivada $c^{\prime \prime}(s)$ é a energia de suavização do contorno que tende a deixá-lo uniforme (energia de curvatura). Já a energia externa $\left(E_{\text {ext }}\right)$, é definida por propriedades da imagem como o gradiente ou intensidade dos pixels.

Dentre vários métodos de Contorno Ativos, este trabalho concentrou-se no MCA Balão, pois de acordo com RIBEIRO et al. [14] este método demonstrou-se rápido e preciso para a aplicação em imagens de diluição na soldagem. Além disso, o MCA Balão apresenta a vantagem em relação ao Snakes tradicional de que não existe a necessidade de inicialização próxima às bordas, no entanto, é preciso inicializa-lo no interior do objeto a ser segmentado.

O Método de Contorno Ativo Balão foi desenvolvido por COHEN e COHEN [17], e define a energia interna como,

$$
\mathrm{E}_{\text {int }}(\mathrm{s})=\alpha F_{\text {cont }}(\mathrm{s})+\beta F_{\text {bal }}(\mathrm{s}) \text {, }
$$

em que $F_{\text {cont }}(\mathrm{s})$, trata-se da força de continuidade e $F_{b a l}(\mathrm{~s})$, a força balão. Os parâmetros $\alpha$ e $\beta$ são pesos de cada força. A força balão possui como objetivo expandir o contorno e pode ser descrita matematicamente como:

$$
F_{b a l}[\mathrm{c}(\mathrm{s})]=\sqrt{E_{x}^{2}+E_{y}^{2}}
$$

em que as componentes Ex e Ey são definidas por:

$$
E_{x}=\left|x(s)-x_{t}\right| \text {, }
$$

$\mathrm{e}$

$$
E_{y}=\left|y(s)-y_{t}\right|,
$$


em que $\mathrm{x}(\mathrm{s})$ e $\mathrm{y}(\mathrm{s})$ são as coordenadas do ponto c(s) onde $F_{b a l}(\mathrm{~s})$ está sendo calculada, $x_{t}$ e $y_{t}$ são as coordenada do centroide da curva. Para implementar o método de Contorno Ativo Balão, MACKIEWICH [18] propôs uma forma que se baseia no centroide do contorno. Posteriormente é preciso normalizar o comprimento entre os pontos do contorno em relação à distância em questão.

A Figura 2 apresenta o funcionamento do MCA Balão aplicado em imagens de materiais soldados capturadas por um estereoscópio. No sistema proposto, inicialmente é necessário definir o ponto de inicialização do método, que é representado pelo ponto vermelho na Figura 2(a). Em sequência, uma curva é gerada automaticamente capaz de se deformar através da minimização das energias vinculadas ao MCA Balão, Figuras 2(b) e 2(c). E a Figura 2(d) apresenta o resultado da segmentação da região de interesse.

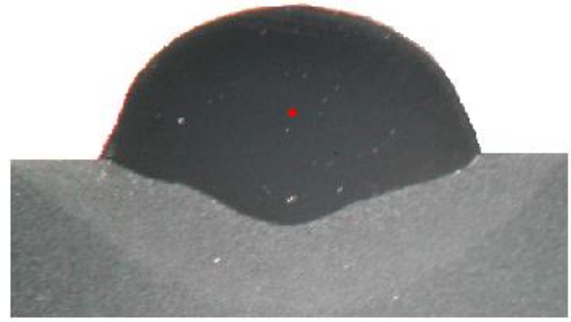

a)

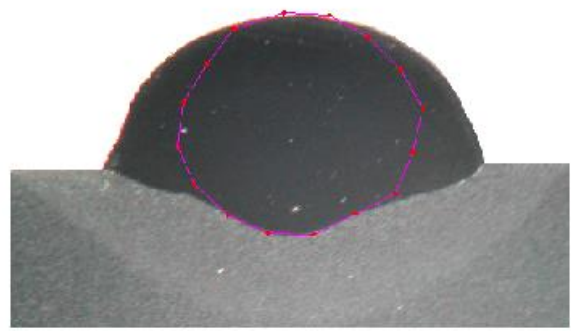

c)

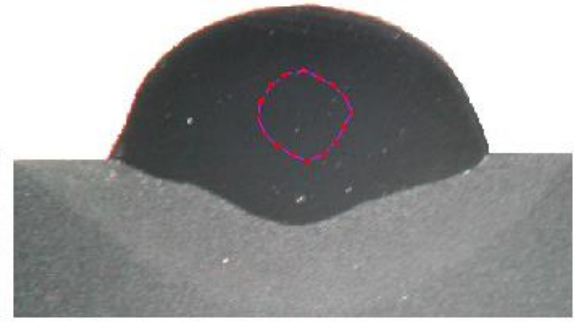

b)

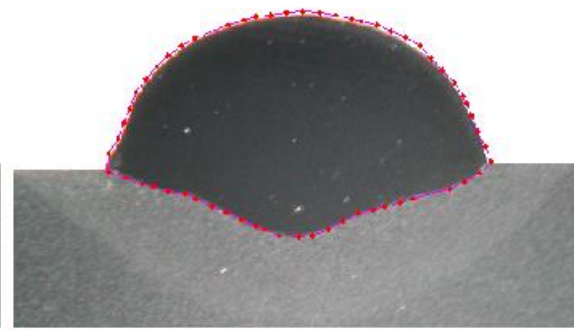

d)

Figura 2: a) Imagem original com o ponto de inicialização definido, b) e c) representam as etapas intermediárias da segmentação e d) é o resultado final da segmentação.

\subsection{Cálculo dos pontos de inflexão da curva}

A determinação de ponto de inflexão está usualmente associada a uma mudança do sentido da concavidade (para cima ou para baixo) da curva de uma função à esquerda e à direita desse ponto, isto é, uma função c tem uma inflexão para $\mathrm{x}=\mathrm{s}$, ou no ponto $(\mathrm{s}, \mathrm{c}(\mathrm{s})$ ), se no ponto $(\mathrm{s}, \mathrm{c}(\mathrm{s}))$ se verifica a mudança do sentido de concavidade da curva [19].

Para a determinação discreta dos pontos c(s) de inflexão da curva gerada pelo MCA Balão, inicialmente é necessário definir as tangentes em relação aos pontos c(s-1) e c(s+1) que são definidas respectivamente nas Equações 6 e 7. Deste modo, obtém-se a inclinação da curva nestes pontos.

$$
\begin{aligned}
& \tan \gamma=\frac{y(s)-y(s-1)}{x(s)-x(s-1)} \\
& \tan \theta=\frac{y(s)-y(s+1)}{x(s)-x(s+1)}
\end{aligned}
$$

Em sequência para definir os pontos de inflexão criou-se uma variável chamada de I(s), que assume resultado binário de acordo as duas propriedades abaixo representadas na Equação 8:

$$
\left\{\begin{array}{l}
\tan \gamma * \tan \theta \geq 0, I(s)=0 \\
\tan \gamma * \tan \theta<0, I(s)=1
\end{array}\right.
$$


Desta forma, quando $\tan \gamma * \tan \theta>0$, implica que os sinais que representam a inclinação da reta do ponto atual em relação respectivamente ao seu ponto anterior e posterior são iguais, então não ocorre mudança na inclinação da curva. Se $\tan \gamma * \tan \theta=0$, significa que $\tan \gamma=0$ e/ou $\tan \theta=0$, então não ocorre variação do sinal da curva. Porém, se $\tan \gamma * \tan \theta<0$ significa que os sinais que representam a inclinação da curva são diferentes, assim definindo o ponto como de inflexão.

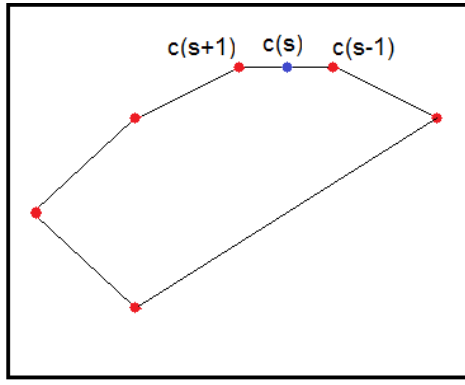

a)

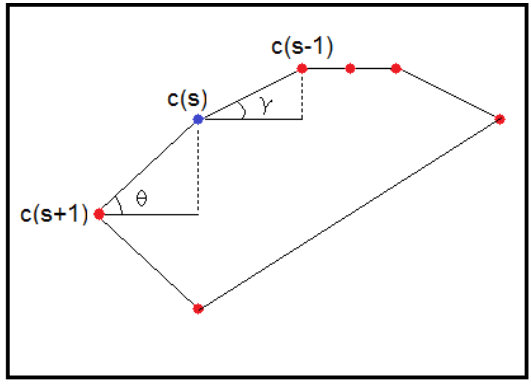

b)

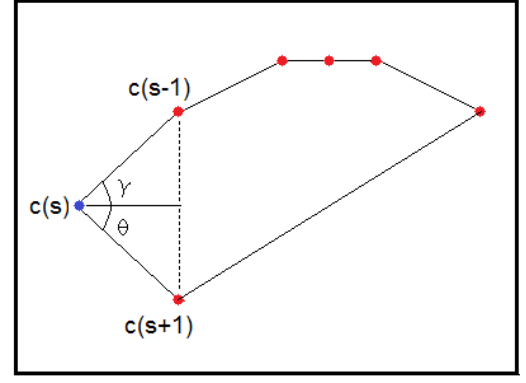

c)

Figura 3: a) e b) representam casos em que não ocorre mudança no sinal da curva e c) representa caso em que ocorre a variação do sinal da curva.

A Figura 3 apresenta três casos mais comuns no cálculo dos pontos de inflexão da curva, e em cada uma o ponto analisado é mostrado em azul. Na Figura 3(a), é verificado que não ocorre variação da inclinação da curva devido que as retas relacionadas ao ponto c(s) não possuem inclinação. A Figura 3(b), apresenta o caso em que a inclinação das retas permanece a mesma. Já na Figura 3(c), apresenta o caso em que o ponto c(s) teve a modificação do sinal da curva, pois a reta entre c(s) e c(s-1) teve o sinal de inclinação diferente da reta relacionada a $\mathrm{c}(\mathrm{s})$ e $\mathrm{c}(\mathrm{s}+1)$.

Em seguida, após a definição dos dois pontos de inflexão da curva gerada pelo MCA Balão, é gerado uma linha que interliga esses pontos afim de construir uma linha de separação entre a região do metal de base fundido e a região do metal de enchimento. A Figura 4, apresenta a construção dessa linha de separação proposta neste trabalho.

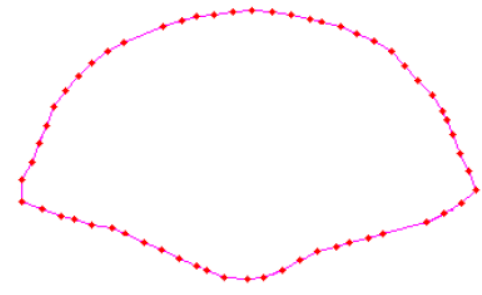

a)

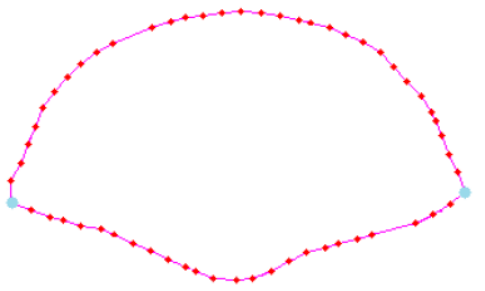

b)

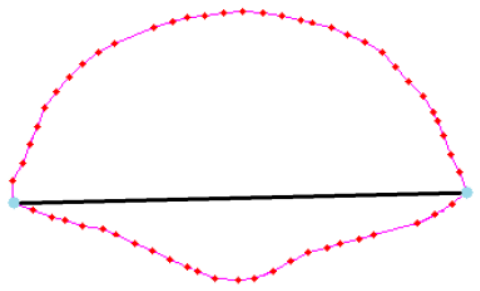

c)

Figura 4: a) Contorno obtido pelo MCA Balão, b) apresentação dos pontos de inflexão do contorno e c) construção da linha de separação de separação entre a região o metal de base fundido e a região do metal de enchimento.

\subsection{Cálculo da taxa de diluição}

A taxa de diluição na soldagem, é uma medida de análise percentual representada pela razão entre a massa fundida do metal de base sobre a massa total do cordão de solda, que pode ser medida pela relação entre as áreas correspondentes.

Geralmente em aplicações que envolvem soldagem de revestimento é necessário realizar a minimização da taxa de diluição, que pode ser feita através das modificações dos parâmetros de soldagem como a velocidade de avanço, a corrente utilizada, o eletrodo aplicado e entre outras [12].

Deste modo, faz-se necessário calcular a taxa de diluição através de imagens adquiridas por um estereoscópio. A Figura 5, mostra as duas regiões que são separadas pela linha gerada a partir dos pontos de inflexão da curva calculados anteriormente, em que a região preta é a Área de Metal de Enchimento (AME) e a região vermelha representa a Área de Penetração (AP). 
Em seguida, a Equação 9 apresenta como é calculado matematicamente a taxa de diluição [14]. Para isso, primeiro é necessário encontrar a Área de Penetração, e posteriormente calcula-se a área total que corresponde ao somatório entre as regiões AME e AP. Por fim, o resultado é dado pela razão entre essas áreas que pode ser visualizada na Equação 9.

Neste trabalho, para o método proposto, foi realizada uma única medição da taxa de diluição para cada amostra. Esta medição única ocorre, pois o sistema computacional desenvolvido retorna valor igual de diluição para a mesma imagem independente de quantas medições sejam realizadas.

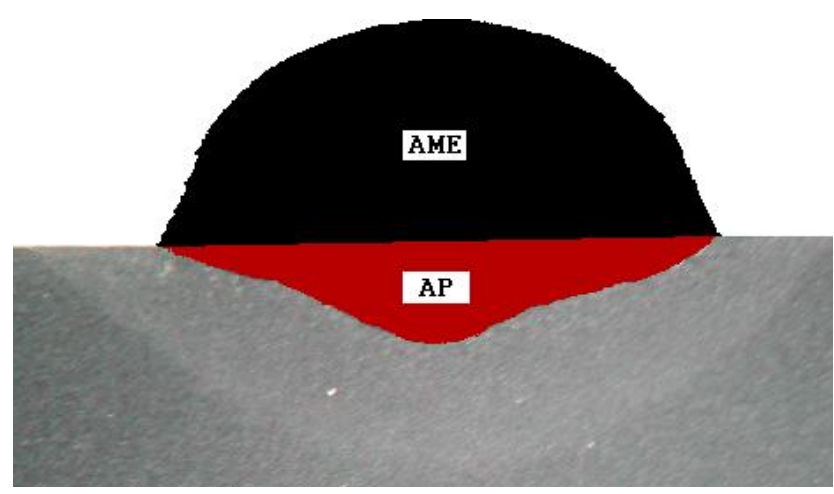

Figura 5: Representação da Área do Metal de Enchimento (AME), na cor preta e a Área de Penetração (AP), na cor vermelha.

$$
\text { Taxa de Diluição }=\frac{\text { Área de Penetração }}{\text { Área de Penetração + Área do Metal de Enchimento }}
$$

\subsection{Medidas de avaliação de desempenho}

Para calcular as métricas de desempenho relativas a qualidade da segmentação foi obtido o padrão-ouro das imagens, que consiste em um especialista realizar a marcação da região correspondente ao metal de solda para cada uma das imagens. Em seguida, foi aplicado o MCA Balão nas mesmas imagens afim de comparar com os resultados de segmentação manual.

Os resultados apresentados nesta etapa são baseados nos pixels da imagem. Desta forma, cada pixel é definido em uma das categorias seguintes, Verdadeiro Positivos (VP) são os pixels que constam no padrão ouro como solda e que são segmentados como solda pelo algoritmo. Verdadeiro Negativos (VN) que consistem em pixels que não constam no padrão ouro como solda e não são segmentados como solda pelo algoritmo. Falso Positivos (FP) que consistem em pixels que não constam no padrão ouro como solda e são segmentados como solda pelo algoritmo. Falso Negativos (FN) que consistem em pixels que constam no padrão ouro como solda e não são segmentados como solda pelo algoritmo.

Por fim, para a etapa de segmentação, foram calculadas as métricas Coeficiente de Similaridade (CS), Sensibilidade (S) e Especificidade (Es), matematicamente representadas pelas equações 10, 11 e 12, respectivamente.

$$
\text { Coeficiente de Similaridade }=\frac{V P+V N}{V P+V N+F P+F N}
$$

$$
\begin{aligned}
& \text { Sensibilidade }=\frac{V P}{V P+F N} \\
& \text { Especificidade }=\frac{V N}{V N+F P}
\end{aligned}
$$


Para a análise dos pontos que definem a linha de separação, foi calculada a distância Euclidiana entre os pontos determinados pelo cálculo da inflexão em relação aos pontos determinados manualmente a fim de verificar o quanto os pontos estão próximos.

\section{RESULTADOS}

Nesta seção, são apresentados os resultados obtidos nas etapas de segmentação, identificação dos pontos de inflexão e cálculo da taxa de diluição. Para melhor compreensão, cada etapa é avaliada separadamente analisando a precisão e o tempo de processamento de cada abordagem. Para esta análise, foi utilizado um banco composto por 37 imagens compostas por diferentes tipos de aços com depósito simples. Essas imagens foram obtidas através de um estereoscópio e foi operado por um especialista. A resolução das imagens capturadas foram de 1280x720 pixels. Os testes foram realizados em um computador com processador Intel Core i5 de 2,4 GHz, 8 GB de RAM e sistema operacional Mac X El Captain 10.11.2. O método computacional proposto, foi desenvolvido na linguagem de programação $\mathrm{C}++$.

\subsection{Resultados da etapa de Segmentação}

O método de segmentação utilizado nesse trabalho foi o Método de Contornos Ativos Balão, que foi responsável pela determinação automática da região correspondente ao metal de solda nas imagens capturadas. $\mathrm{O}$ MCA Balão, utilizou o kernel 5 e teve o ponto de inicialização indicado por um especialista. O parâmetro de parada do método foi baseado na estabilização do perímetro do contorno.

As energias envolvidas em um modelo paramétrico de contorno ativo precisam ser parametrizadas e cada componente da energia tem uma importância diferente no cálculo da energia total. Como tal, os parâmetros $\alpha$, $\beta$ e $\tau$ definem, respectivamente, os pesos da força balão, da força de continuidade e da energia externa, respectivamente, no cálculo da energia total. Os parâmetros ótimos foram definidos pela validação cruzada variando cada parâmetro e considerando a soma de todos como igual a 1 (um). Para esta aplicação, a configuração ótima obtida foi $\alpha=0,02 \operatorname{com} \beta=0,08$ e $\tau=0,9$. Esta opção levará ao menor tempo de processamento e maior eficiência possíveis.

A seguir a Figura 6, apresenta o resultado do Coeficiente de Similaridade, Sensibilidade e Especificidade da segmentação proposta em relação a segmentação manual. Vale ressaltar que, em cada gráfico, foi estabelecida uma escala diferente a fim de apresentar com maior definição os resultados obtidos. 


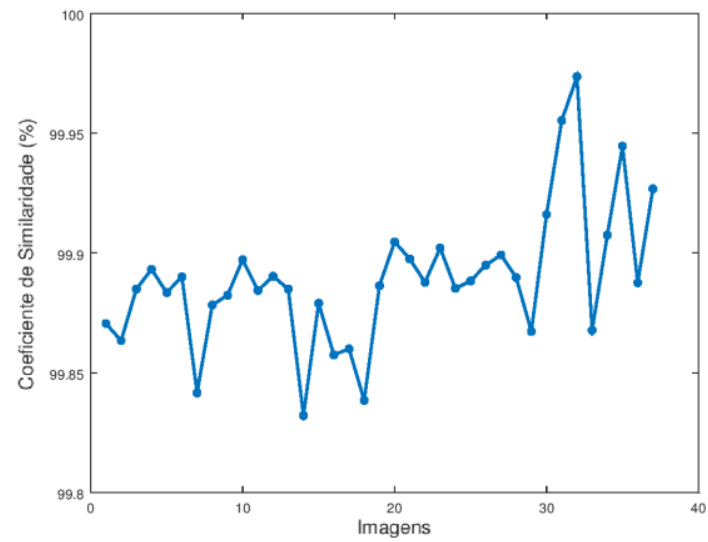

a)

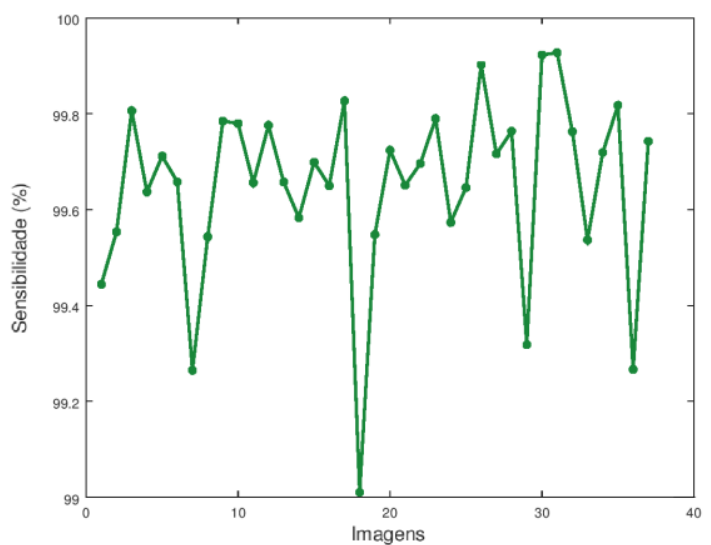

b)

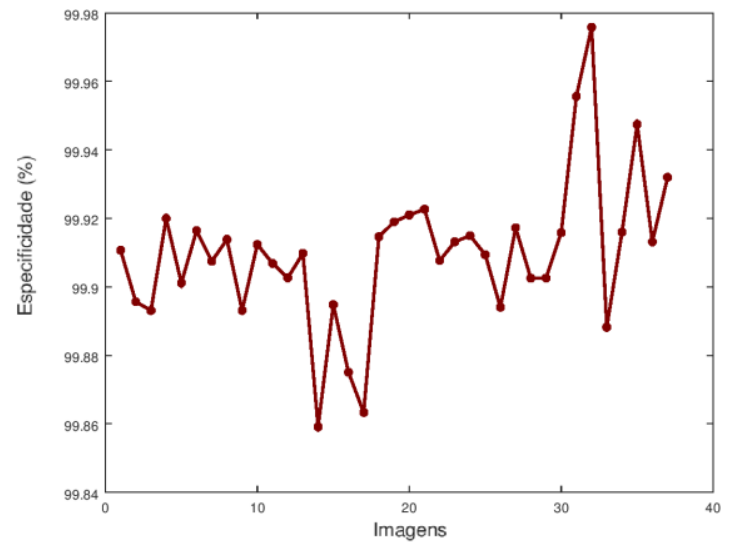

c)

Figura 6: Resultados da segmentação proposta em relação a segmentação manual para cada uma das 37 amostras onde a) representa o Coeficiente de Similaridade (CS), b) a Sensibilidade (S) e c) a Especificidade (Es).

Outra avaliação realizada foi quanto ao tempo gasto para a segmentação. Ao utilizar o método tradicional, o tempo médio para segmentação manual da área de solda em cada amostra foi de $323 \pm 1,34$ segundos. Como no trabalho o processo é repetido em 37 amostras, tem-se um tempo total de quase 4 horas para realizar essa segmentação manual. Na abordagem proposta obteve-se um tempo médio de processamento de $4 \pm$ 0,63 segundos para cada amostra, e um tempo total menor que 3 minutos para analisar todo o banco de imagens disponível.

\subsection{Resultados da identificação dos pontos de inflexão}

Para realizar a avaliação da definição automática dos pontos de inflexão da curva, inicialmente foi necessário que um especialista marcasse de forma manual esses pontos. Em seguida, foi aplicado o algoritmo para calcular de forma automática os pontos de inflexão da curva, obtidos através das curvas construídas a partir do MCA Balão.

Na Figura 7, é apresentado o quanto os pontos selecionados automaticamente pela metodologia proposta se distanciam dos pontos identificados manualmente pelo especialista, através do cálculo da distância Euclidiana. 


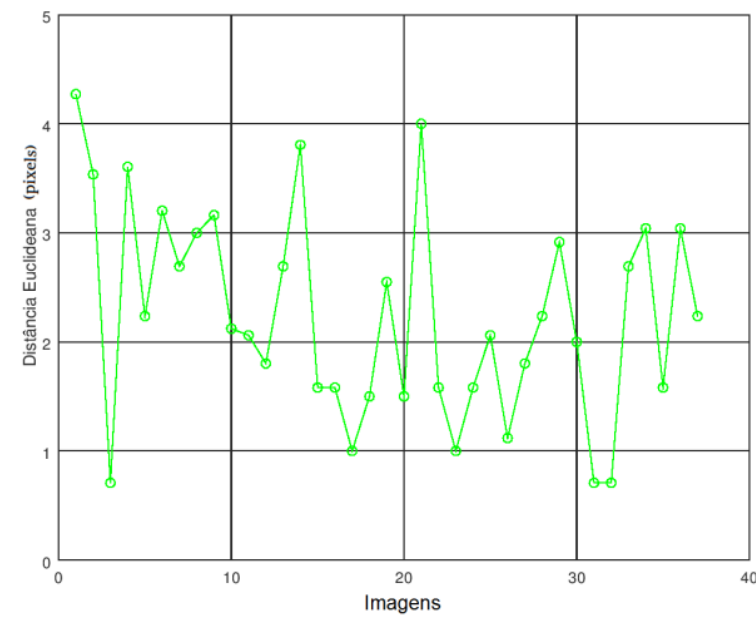

a)

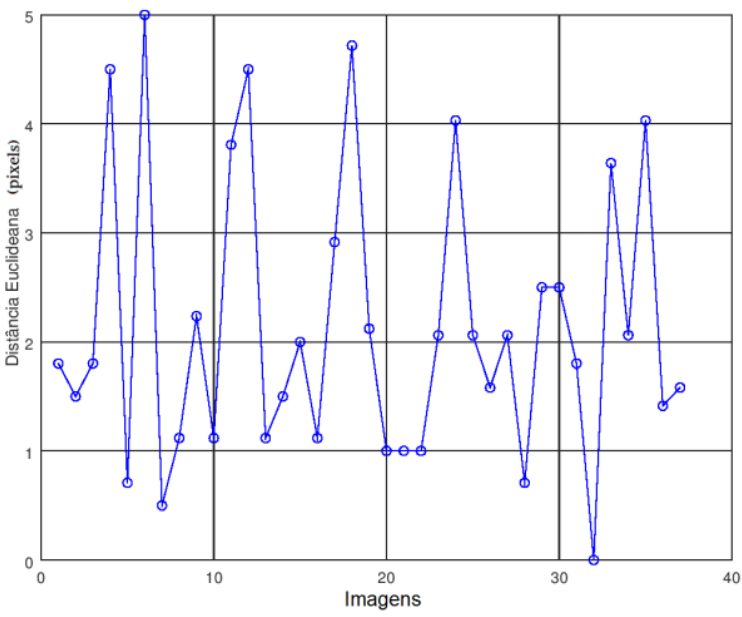

b)

Figura 7: Distância Euclidiana entre os pontos manualmente definidos e os pontos identificados pela inflexão da curva, em que a) são os pontos localizados no lado extremo esquerdo da solda e b) são os pontos localizados no lado extremo direito da solda.

\subsection{Resultados do Cálculo da Taxa de Diluição}

Para validar as taxas de diluição obtidas pela metodologia proposta, foi necessário realizar uma comparação numérica dos resultados com o método manual, e com um método já estabelecido na literatura, proposto por RIBEIRO et al. [14]. O sistema desenvolvido em [14] é semiautomático, e foi escolhido com o objetivo de avaliar de maneira mais ampla a metodologia proposta, pois também utiliza os contornos ativos para o cálculo da taxa de diluição.

No método manual, a taxa de diluição foi calculada pela definição das áreas correspondentes ao metal de enchimento adicionado e ao metal de base fundido, através do trabalho manual de um especialista apenas auxiliado por um programa de desenho.

Para o sistema semiautomático, proposto por [14], foram calculadas as áreas do metal de enchimento adicionado e do metal de base fundido, através de um especialista que definiu três pontos chaves na imagem. Dentre os três pontos, um é encarregado de inicializar o contorno dentro da solda e os outros dois definem a linha de separação entre as áreas a serem calculadas.

A metodologia proposta neste trabalho apenas necessitou definir um ponto interno a área de solda para inicializar o MCA Balão. E em seguida, de forma automática é encontrada a linha de separação da solda a partir do cálculo dos pontos de inflexão do contorno. Desta forma, é determinado automaticamente as áreas do metal de enchimento adicionado e do metal de base fundido.

A Tabela 1, apresenta os resultados do modelo proposto em que cálculo manual é representado por (CM), cálculo proposto por (CP) e o cálculo semiautomático por (CS).

Tabela 1: Valores da taxa de diluição para cada amostra pelo cálculo manual $(\mathrm{CM})$, cálculo proposto $(\mathrm{CP})$ e o cálculo semiautomático (CS) com a determinação dos erros percentuais relativos.

\begin{tabular}{c|ccccc}
\hline Faixa de diluição & Manual & Semiautomático & Proposto & Erro \% entre CM e CP & Erro \% entre CS e CP \\
\hline & 15,1063 & 13,9487 & 14,1885 & 6,07496 & 1,71943 \\
& 16,4001 & 16,1353 & 15,9165 & 2,94818 & 1,3557 \\
$15-25$ & 18,7512 & 18,9294 & 18,8811 & 0,69303 & 0,25508 \\
& 19,1144 & 19,8976 & 19,9644 & 4,44711 & 0,33573 \\
& 19,4064 & 18,9282 & 18,9606 & 2,29693 & 0,17102 \\
& 20,1323 & 19,4997 & 19,7263 & 2,01628 & 1,16208 \\
& 20,2143 & 20,9301 & 19,702 & 2,53431 & 5,86771
\end{tabular}




\begin{tabular}{|c|c|c|c|c|c|}
\hline & 20,6501 & 20,2634 & 19,955 & 3,36549 & 1,52224 \\
\hline & 20,7353 & 20,21 & 19,8301 & 4,36535 & 1,88003 \\
\hline & 21,6276 & 20,9303 & 20,5336 & 5,05801 & 1,8954 \\
\hline & 21,9939 & 21,6322 & 21,9035 & 0,41074 & 1,25419 \\
\hline \multirow{16}{*}{$25-35$} & 26,6452 & 26,7396 & 26,5595 & 0,32132 & 0,67325 \\
\hline & 27,0603 & 25,7848 & 26,5166 & 2,00892 & 2,83814 \\
\hline & 28,5487 & 27,4882 & 27,0578 & 5,22226 & 1,5659 \\
\hline & 28,5565 & 28,5121 & 27,1136 & 5,05271 & 4,90508 \\
\hline & 28,806 & 28,0337 & 27,488 & 4,57517 & 1,94658 \\
\hline & 28,9548 & 28,9434 & 28,7762 & 0,61665 & 0,57769 \\
\hline & 30,3521 & 29,1039 & 29,4606 & 2,93695 & 1,22584 \\
\hline & 30,4028 & 30,3319 & 30,1681 & 0,77187 & 0,54003 \\
\hline & 30,9445 & 30,0099 & 29,8961 & 3,38769 & 0,37919 \\
\hline & 32,8943 & 33,3957 & 33,5725 & 2,06184 & 0,52922 \\
\hline & 33,3371 & 32,9184 & 33,092 & 0,73519 & 0,52712 \\
\hline & 33,3735 & 32,9106 & 34,1011 & 2,18023 & 3,61724 \\
\hline & 33,3915 & 32,1009 & 32,2941 & 3,2864 & 0,60184 \\
\hline & 34,0359 & 34,6811 & 34,5668 & 1,56001 & 0,32964 \\
\hline & 34,5851 & 33,7848 & 33,8697 & 2,06829 & 0,25128 \\
\hline & 34,7563 & 33,7024 & 34,2123 & 1,56493 & 1,51309 \\
\hline \multirow{10}{*}{$35-50$} & 35,4162 & 34,8078 & 34,8847 & 1,50066 & 0,22084 \\
\hline & 41,7323 & 41,6017 & 41,4603 & 0,65166 & 0,33978 \\
\hline & 41,7414 & 42,5253 & 42,2936 & 1,32301 & 0,54483 \\
\hline & 41,9844 & 40,6942 & 41,0268 & 2,28068 & 0,81742 \\
\hline & 43,353 & 43,1786 & 43,0704 & 0,6517 & 0,25049 \\
\hline & 44,5043 & 42,9434 & 43,2919 & 2,72404 & 0,81168 \\
\hline & 45,1987 & 44,6745 & 44,1359 & 2,35132 & 1,20559 \\
\hline & 47,8402 & 46,0102 & 46,3736 & 3,06554 & 0,7899 \\
\hline & 48,6782 & 48,6689 & 48,8549 & 0,36317 & 0,38217 \\
\hline & 49,8012 & 49,2793 & 48,8072 & 1,99591 & 0,95805 \\
\hline
\end{tabular}

\section{DISCUSSÃO}

Inicialmente, é importante analisar os resultados obtidos pela etapa de segmentação, pois esta tem grande influência sobre as próximas fases do sistema proposto. Para isto, a Figura 6 apresenta os resultados das métricas Coeficiente de Similaridade, Sensibilidade e Especificidade. Analisando os resultados, foi verificado que a segmentação proposta demostrou valores sempre superiores a $99,00 \%$ em todas as métricas e em alguns momentos atingindo $99,97 \%$. Além disso, o tempo médio de segmentação realizado pelo MCA Balão para cada imagem foi de $4 \pm 0,63$ segundos, pelo método convencional foi de $323 \pm 1,34$ segundos e pelo semiautomático proposto por [14] foi de 6,7 $\pm 0,81$. Assim, avaliando de uma forma geral a segmentação proposta tem acerto equivalente ao método manual, e é aproximadamente 80x mais rápida que o método convencional e 1,675x mais rápido que o método semiautomático.

Em seguida, o cálculo dos pontos de inflexão, a partir do contorno gerado pelo MCA Balão, possibilitou a construção da linha de separação entre o metal de enchimento adicionado e o metal de base fundido. Porém, a Figura 8(a) apresenta um caso em que o formato do contorno, gera um ponto de inflexão desnecessário para a construção da linha de separação. Para resolver essa falha, foi realizado uma filtragem dos pontos de inflexão baseada em buscar apenas os dois pontos com menor e maior coordenada. E posteriormente a 
linha de separação é construída de maneira correta como visualizado na Figura 8(c), pois essa filtragem retorna apenas os dois pontos necessários que são mostrados na Figura 8(b).

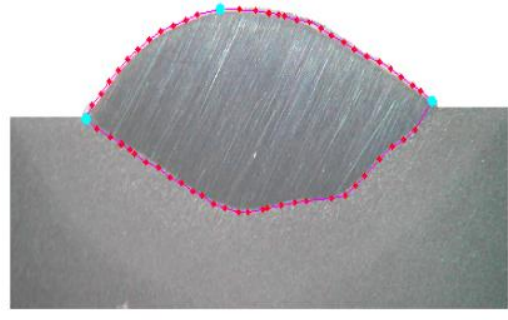

a)

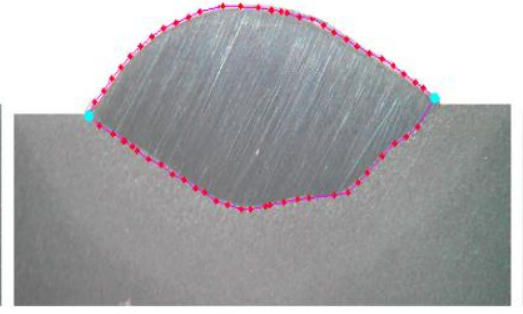

b)

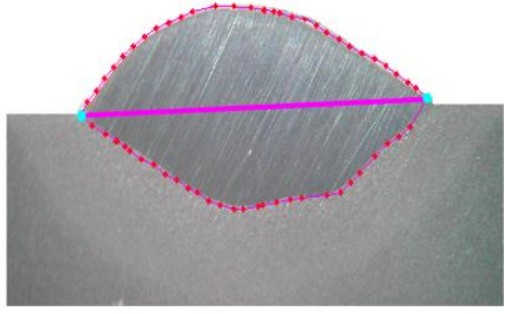

c)

Figura 8: a) Todos os pontos de inflexão calculados, b) pontos de inflexão após a filtragem e c) construção da linha de separação.

Após essa constatação experimental, é possível avaliar a exatidão dos pontos de inflexão obtidos pela metodologia proposta. Para isto, a análise da Figura 7 permite verificar-se a distância Euclidiana entre os pontos definidos por um especialista e os pontos identificados pela inflexão do contorno obtido. É aferido que tantos os pontos da direita quanto os pontos da esquerda variam no máximo uma distância de 5 pixels. Além disso, apresentam uma distância média de $2,19 \pm 0,91$. Isso implica, que os pontos identificados pelo sistema proposto demonstram equivalência em relação aos pontos marcados pelo especialista, pois essa distância observada é irrelevante se comparada com o tamanho original da imagem.

Por fim, a Tabela 1 apresenta os resultados da taxa de diluição para cada amostra e demonstra o erro relativo entre os métodos. Foi verificado que o erro percentual atribuído entre o cálculo manual e o proposto atingiu no máximo $6,07 \%$. E o erro relativo entre o cálculo realizado pelo método semiautomático e o proposto foi no máximo de $5,86 \%$. Além disso, algumas vezes o erro relativo é menor que $1 \%$. Com isso, foi constatado a equivalência da metodologia proposta com os outros métodos comparados, pois ocorre pouca modificação numérica do valor da taxa de diluição.

Quanto ao tempo de análise total, o método proposto apresenta grande vantagem, visto que demora em média aproximadamente 5 segundos para cada imagem, que totalizam 185 segundos para todas as imagens, enquanto o método convencional demora aproximadamente 14856 segundos para todas as imagens. Isso, implica que o método proposto é bastante rápido, mesmo em casos com muitas amostras. Vale à pena ressaltar que as imagens utilizadas possuem tamanho de 1280x720, e caso fossem usadas imagens com maior resolução, este tempo iria aumentar na mesma proporção da imagem.

\section{CONCLUSÕES}

A medição da taxa de diluição pelo método tradicional é caracterizada por ser um processo manual, exaustivo e muitas vezes propenso ao erro devido à fadiga do operador após análise de muitas imagens. Com o intuito de aprimorar o processo de medição, foi desenvolvido um sistema de visão computacional para realizar esta operação de forma automática.

Em relação as tecnologias utilizadas, o método MCA Balão apresentou acurácia média igual a 99,89 \pm 0,019 e tempo de processamento médio igual a $4 \pm 0,63$ segundos. Na etapa do cálculo dos pontos de inflexão, que definem a linha de separação entre as áreas de interesse, a distância média entre o ponto determinado por um especialista na área de soldagem e o ponto definido pelo sistema proposto é de apenas 2,19 $\pm 0,91$ pixels. Já na etapa de medição da taxa de diluição, o erro médio relativo foi de $1,83 \pm 1,21 \%$ com relação ao método tradicional. Com isto, têm-se que o sistema desenvolvido para esta aplicação atinge a qualidade equivalente a outros métodos, porém se destaca por ser 80x mais rápido do que o método tradicional.

Assim, este artigo propõe uma nova abordagem automática para o cálculo da taxa de diluição em imagens digitais através do Método de Contornos Ativos Balão. A principal contribuição do sistema desenvolvido é a determinação automática dos pontos que definem a linha de separação entre a região do metal de base fundido e a região com metal de enchimento através do cálculo de inflexão da curva. A partir dos resultados obtidos pelo sistema proposto, foram feitas comparações com métodos já estabelecidos na literatura, apresentando assim sua superioridade nesta aplicação. 
Com o intuito de aumentar a robustez do sistema desenvolvido, estuda-se em trabalhos futuros a inclusão de diagramas de composição, tal como o diagrama de Schaeffler, empregado na análise da soldagem de aços inoxidáveis, a fim de que baseado no cálculo da diluição o sistema desenvolvido informe a composição da liga. Outro ponto a ser considerado em trabalhos futuros é a utilização de amostras com diluições mais baixas a fim de verificar se o sistema desenvolvido é eficiente para a diferente geometria do cordão encontrada nestes casos, assim como, os pontos de inflexão mais atenuados.

\section{AGRADECIMENTOS}

Os autores agradecem ao apoio financeiro do IFCE via editais de fomento PROAPP-IFCE de 2014, PAPEX/IFCE de 2015 e PROFEP/IFCE-Maracanaú de 2016.

\section{BIBLIOGRAFIA}

[1] REBOUÇAS FILHO, P.P., MOREIRA, F.D.L., XAVIER, F.G.L., et al., "New analysis method application in metallographic images through the construction of mosaics via speeded up robust features and scale invariant feature transform", Materials, v. 8, n. 7, pp. 3864-3882, Jun. 2015.

[2] ALBUQUERQUE, V.H.C., BARBOSA, C.V., SILVA, C.C., et al., "Ultrasonic sensor signals and optimum path forest classifier for the microstructural characterization of thermally-aged Inconel 625 alloy", Sensors, v. 15, n. 6, pp. 12474-12497, May 2015.

[3] PAPA, J.P., NAKAMURA, R.Y.M., ALBUQUERQUE, V.H.C., et al., "Computer techniques towards the automatic characterization of graphite particles in metallographic images of industrial materials", Expert Systems with Applications, v. 40, n. 2, pp. 590-597, Jul. 2013.

[4] PAPA, J.P., ALBUQUERQUE, V.H.C., FALCÃO, A.X., et al., "Fast automatic microstructural segmentation of ferrous alloy samples using optimum-path Forest”, In: Computational Modeling of Objects Represented in Images, pp. 210-220, Buffalo, New York, USA, May 2010.

[5] REBOUÇAS, E.S., BRAGA, A .M., MARQUES, R.C.P., et al., "A new approach to calculate the nodule density of ductile cast iron graphite using a level set”, Measurement, v. 89, n. 2, pp. 316-321, Apr. 2016.

[6] PEIXOTO, F.M.A., REBOUÇAS, E.S., XAVIER, F.G.L., et al., "Desenvolvimento de um Software para cálculo da densidade de nódulos de grafita em ferro fundido nodular através de Processamento Digital de Imagens", Matéria (UFRJ), v. 20, n. 1, pp. 262-272, Mar. 2015.

[7] ALBUQUERQUE, V.H.C., CORTEZ, P.C., ALEXANDRIA, A.R., et al., "A new solution for automatic microstructures analysis from images based on a backpropagation artificial neural network", Nondestructive Testing and Evaluation, v. 23, n. 4, pp. 273-283, Jun. 2008.

[8] ALBUQUERQUE, V.H.C., TAVARES, J.M.R.S., CORTEZ, P.C., et al., "Quantification of the microstructures of hypoeutectic white cast iron using mathematical morphology and an artificial neural network", International Journal of Microstructure and Materials Properties, v. 5, n. 1, pp. 52-64, Jun. 2010.

[9] ALBUQUERQUE, V.H.C., NAKAMURA, R.Y.M., PAPA, J.P., et al., "Automatic segmentation of the secondary austenite-phase island precipitates in a super duplex stainless steel weld metal", In: III ECCOMAS Thematic Conference on Computational Vision and Medical Image Processing: Vip IMAGE 2011, pp. 161166, Olhão, Algarve, Portugal, Oct. 2011.

[10] ALBUQUERQUE, V.H.C., ALEXANDRIA, A.R., CORTEZ, P.C., et al., "Evaluation of multilayer perceptron and self-organizing map neural network topologies applied on microstructure segmentation from metallographic images", NDT \& E International, v. 42, n. 7, pp. 644-651, May 2009.

[11] WAINER, E., BRANDI, S., MELLO, F., Soldagem-Processos e Metalurgia, 19 ed. Editora Edgard Blucher, 1992.

[12] NADEZHDIN, A.D., DAVISON, T., "Effects of Weld Dilution on Digester Weld Overlay", In: 11th International Symposium on Corrosion in the Pulp and Paper Industry, 2004.

[13] REBOUÇAS FILHO, P.P., CAVALCANTE, T.S., ALBUQUERQUE, V.H.C., et al., "Sistema Computacional para Determinação da Taxa de Diluição em Soldadura", Revista da Associação Portuguesa de Análise Experimental de Tensões, v. 18, pp. 55-61, 2010. 
[14] RIBEIRO, A.B.N., CAVALCANTE, T.S, REBOUÇAS FILHO, P.P., "Desenvolvimento de Programa Computacional para Medição Semi-automática de Altura, Largura e Profundidade do Cordão de Solda, e Taxa de Diluição", In: VI Congresso Nacional de Engenharia Mecânica, 2010.

[15] GONZALEZ, R.C., WOODS, R.E., Digital Image Processing, 3 ed., São Paulo, Pearson, 2008.

[16] KASS, M., WITKIN, A., TERZOPOULOS, D., "Snakes: active contours models". International Journal of Computer Vision, v. 1, n. 4, pp. 321-331, 1987.

[17] COHEN, L.D., COHEN, I., "Finite-element for active contours models and Balloons for 2d and 3d images", IEEE transaction on Pattern analysis and Machine Intelligence, v. 15, n. 11, pp. 1131 - 1147. Nov 1993.

[18] MACKIEWICH, B., Intracranial boundary detection and radio frequency correction in magnetic resonance images. Master's thesis, Simon Fraser University, Computer Science Department, Burnaby, 1995.

[19] GUIDORIZZI, H.L., Um Curso de Cálculo - Volume I, 5 ed., Rio de Janeiro. LTC - Livros Técnicos e Científicos, 2001.

\section{ORCID}

Gabriel Bandeira Holanda

https://orcid.org/0000-0001-7850-8136

Daniel de Alencar Lima

https://orcid.org/0000-0001-9914-8694

Pedro Pedrosa Rebouças Filho

https://orcid.org/0000-0002-1878-5489 